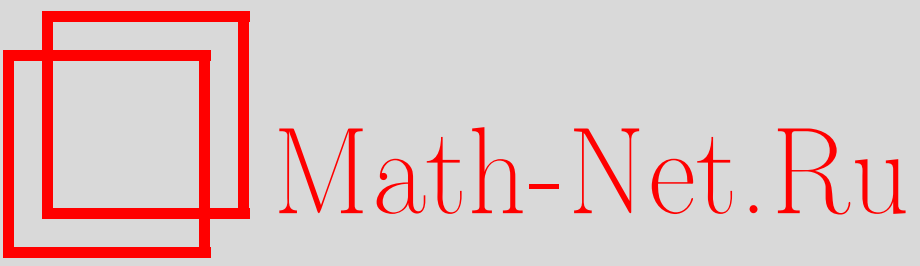

А. Н. Лезнов, Двумерные интегрируемые ультра-Тода-отображения и цепочки, TMФ, 1998, том 117, номер 1, 107-122

DOI: https://doi.org/10.4213/tmf920

Использование Общероссийского математического портала Math-Net.Ru подразумевает, что вы прочитали и согласны с пользовательским соглашением

http://www.mathnet.ru/rus/agreement

Параметры загрузки:

IP: 3.85 .7 .115

26 апреля 2023 г., 09:59:27 


\section{ДВУМЕРНЫЕ ИНТЕГРИРУЕМЫЕ УЛЬТРА-ТОДА-ОТОБРАЖЕНИЯ И ЦЕПОЧКИ}

Вводится новый класс двумерных интегрируемых отображений и цепочек. Указан путь построения иерархий $(1+2)$ интегрируемых систем, инвариантных относительно такого рода дискретных преобразований. Получены явные выражения для решений солитонного типа в терминах матричных элементов фундаментальных представлений полупростых алгебр.

\section{1. ВВЕДЕНИЕ: КЛЮЧЕВАЯ РОЛЬ ЦЕПОЧЕК ТОДЫ В ТЕОРИИ ИНТЕГРИРУЕМЫХ СИСТЕМ}

Систему уравнений бесконечной цепочки Тоды можно представить многими эквивалентными способами. Наиболее привычны следующие два:

$$
(\ln v)_{x y}=\frac{\overleftarrow{v}}{v}-\frac{v}{\vec{v}}, \quad(\ln \theta)_{x y}=\overleftarrow{\theta}-2 \theta+\vec{\theta}
$$

где $v \equiv v_{s}, \overleftarrow{v} \equiv v_{s+1}, \vec{v} \equiv v_{s-1}, s-$ натуральное число и $x, y$-независимые аргументы.

Уравнения (1) могут рассматриваться как определение некоторого отображения, посредством которого две начальные функции $v, \vec{v}(\theta, \vec{\theta})$ связаны с двумя конечными $\overleftarrow{v}$, $v(\overleftarrow{\theta}, \theta)$

Мы воспользуемся первой из систем (1), переписав ее в эквивалентной форме:

$$
\overleftarrow{u}=\frac{1}{v}, \quad \overleftarrow{v}=v\left(v u-(\ln v)_{x y}\right)
$$

Замечательное свойство отображения (подстановки) (2) состоит в его интегрируемости (см., например, $[1,2])$. Это означает, что соответствуюшая ему система уравнений симметрии (возникаюшая как результат вариационного дифференцирования (2))

$$
\overleftarrow{U}=-\frac{1}{v^{2}} V, \quad \overleftarrow{V}=v^{2} U+\left(2 u v-(\ln v)_{x y}\right) V-v\left(\frac{V}{v}\right)_{x y}
$$

\footnotetext{
* Институт физики высоких энергий, Протвино, Московская область, Россия
} 
обладает бесконечным набором нетривиальных решений (см., например, [3]). В системе (3) независимыми аргументами функций $U, V$ являются $u, v$ (из (2)) и их производные до определенного порядка по "пространственным" координатам $x, y . \overleftarrow{U}, \overleftarrow{V}$ - те же функции, но от преобразованных в соответствии с (2) аргументов.

Каждое решение (3) связано с интегрируемой в смысле Лиувилля (бесконечное число законов сохранения в инволюции) системой уравнений эволюционного типа

$$
u_{t}=U, \quad v_{t}=V \text {. }
$$

Более того, построенные таким образом системы инвариантны относительно преобразований подстановки (2).

Подавляюшее большинство систем, проинтегрированных к настоящему времени различными методами [4] (в частности, методом обратной задачи рассеяния), напрямую связано с дискретной симметрией Тоды (2) (или с соответствующими преобразованиями Беклунда).

При определенных граничных условиях уравнения бесконечной цепочки Тоды могут обрываться и переходить в конечномерную интегрируемую систему, для которой различные классы решений хорошо изучены. Наиболее известны цепочка Тоды с закрепленными концами: $v_{0}^{-1}=v_{N}=0$, и периодическая цепочка $v_{0}=v_{N}$. В первом случае удается найти обшее решение [5], зависяшее от необходимого количества произвольных функций, а во втором - только многосолитонный подкласс решений, зависяших от сколь угодно большого набора числовых параметров [6]. Обшее решение в этом случае может быть записано в форме бесконечных абсолютно сходящихся рядов [7].

Общее решение для цепочки Тоды с закрепленными концами при $N=2 m$ связано напрямую с $m$-солитонными решениями эволюционных уравнений (4). А именно, решение $u_{m}, v_{m}$ для такой цепочки точно совпадает (при некоторых дополнительных ограничениях на произвольные функции) с $m$-солитонными решениями эволюционных уравнений (4).

Таким образом, уравнения бесконечной цепочки Тоды играют двойственную роль в теории интегрируемых систем: с одной стороны, они определяют явный вид интегрируемой системы (как решение уравнения симметрии), а с другой, решения для "обрезанной” цепочки позволяют построить в явном виде некоторые классы точных решений эволюционных уравнений.

В работах $[1,2]$ была высказана гипотеза, что теория интегрируемых систем эквивалентна теории представлений группы интегрируемых отображений. Цепочка Тоды простейший аргумент, подкрепляющий эту гипотезу.

В настоящей работе вводится новый класс интегрируемых отображений и цепочек. По сравнению с цепочкой Тоды они имеют большее число неизвестных функций в каждом узле решетки. Такие отображения интегрируемы, и им соответствуют иерархии интегрируемых систем, инвариантных по отношению к рассматриваемым ниже дискретным подстановкам. При фиксированных коншах обшие решения выражаются в терминах матричных элементов фундаментальных представлений полупростых алгебр. 


\section{2. ИНТЕГРИРУЕМЫЕ ЦЕПОЧКИ И ГРАДУИРОВАННЫЕ АЛГЕБРЫ}

В работе [8] (см. также монографию [9]) предложен прямой способ построения точно интегрируемых систем на основе произвольной градуированной (супер)алгебры. Ниже мы воспользуемся этим способом применительно к конкретному случаю полупростой алгебры серии $A_{n}$.

Для генераторов простых корней алгебры и соответствующих им элементов Картана используем обозначения $X_{\alpha}^{ \pm}, h_{\alpha}$. Подпространства с градуировочными индексами $\pm s$ строятся из генераторов алгебры $A_{n}$, которые, в свою очередь, могут быть построены из генераторов $s$ простых корней. Основное уравнение [8]

$$
\left[\partial_{x}-\sum_{s=1}^{m_{1}} A^{-s}, \partial_{y}-(\rho h)-\sum_{s=1}^{m_{2}} A^{+s}\right]=0
$$

может быть в данном случае значительно конкретизировано и позволяет записать уравнения цепочки в достаточно обозримом виде.

Генераторы $\pm s$ градуированных подпространств следуюшим образом связаны с генераторами простых корней:

$$
Y_{\alpha}^{+s}=\left[X_{\alpha}^{+}, \ldots\left[X_{\alpha+s-1}^{+}, X_{\alpha+s}^{+}\right] \ldots\right], \quad Y_{\alpha}^{-s}=\left[\ldots\left[X_{\alpha+s}^{-}, X_{\alpha+s-1}^{-}\right] \ldots, X_{\alpha}^{-}\right] .
$$

Как следствие определений (6) возникает следующая система коммутационных соотношений:

$$
\begin{aligned}
& {\left[Y_{\alpha}^{-i}, Y_{\beta}^{+j}\right]=\delta_{\alpha+i, \beta+j} Y_{\alpha}^{-i+j}-\delta_{\alpha, \beta} Y_{\alpha+j}^{-i+j}, \quad i \leqslant j,} \\
& {\left[Y_{\alpha}^{+i}, Y_{\beta}^{+j}\right]=-\delta_{\alpha, \beta+j} Y_{\alpha}^{i+j}+\delta_{\alpha+i, \beta} Y_{\beta}^{i+j},} \\
& {\left[Y_{\alpha}^{-i}, Y_{\beta}^{+i}\right]=\delta_{\alpha, \beta} \sum_{s=0}^{i-1} h_{\alpha+i} .}
\end{aligned}
$$

Далее,

$$
A^{-s}=\sum_{\alpha} Y_{\alpha}^{-s} f_{\alpha}^{s}, \quad A^{+s}=\sum_{\beta} Y_{\beta}^{+s} \bar{f}_{\beta}^{s} .
$$

Используя (7), перепишем основное уравнение (5) в виде

$$
\begin{aligned}
& \left(f_{\alpha}^{s}\right)_{y}+\left(\sum_{k=0}^{s-1} \bar{\rho}_{\alpha+k}\right) f_{\alpha}^{s}-\sum_{k=1}^{m_{1}-s}\left(f_{\alpha}^{s+k} \bar{f}_{\alpha+s}^{k}-f_{\alpha-k}^{s+k} \bar{f}_{\alpha-k}^{k}\right)=0 \\
& \bar{\rho}_{\alpha}=-\rho_{\alpha+1}+2 \rho_{\alpha}-\rho_{\alpha-1}, \quad\left(\rho_{\alpha}\right)_{x}+\sum_{s=1}^{\min \left(m_{1}, m_{2}\right)} \sum_{k=0}^{s-1} f_{\alpha-k}^{s} \bar{f}_{\alpha-k}^{s}=0 \\
& \left(\bar{f}_{\alpha}^{s}\right)_{x}-\sum_{k=1}^{m_{2}-s}\left(\bar{f}_{\alpha}^{s+k} f_{\alpha+s}^{k}-\bar{f}_{\alpha-k}^{s+k} f_{\alpha-k}^{k}\right)=0 .
\end{aligned}
$$

Назовем систему (8) ультра-Тода (УТ)-цепочкой, УТ $\left(m_{1}, m_{2}\right)$, имея в виду, что обычная двумерная цепочка Тоды для серии $A_{n}$ возникает при специальном выборе параметров $m_{1}=m_{2}=1$. Конкретный вычислительньй рецепт, изложенньй в работе [8], позволяет точно проинтегрировать (8). 


\section{3. СИСТЕМА УТ(2,2) И ЕЕ ОБШЕЕ РЕШЕНИЕ}

В случае $m_{1}=m_{2}=2$ в каждом узле изначально имеются 5 неизвестных функций: $\rho_{\alpha}, f_{\alpha}^{1}, \bar{f}_{\alpha}^{1}, f_{\alpha}^{2}, \bar{f}_{\alpha}^{2}$. Однако, вводя калибровочно-инвариантные функции

$$
q_{\alpha}=f_{\alpha}^{1} \bar{f}_{\alpha}^{1}, \quad p_{\alpha}=\frac{f_{\alpha}^{2}}{f_{\alpha}^{1} f_{\alpha+1}^{1}}, \quad \bar{p}_{\alpha}=\frac{\bar{f}_{\alpha}^{2}}{\bar{f}_{\alpha}^{1} \bar{f}_{\alpha+1}^{1}},
$$

мы сокрашаем число неизвестных до трех (в каждом узле) и можем переписать (8) в виде

$$
\begin{aligned}
& (\ln p)_{y}+\left(\overleftarrow{q}^{2} \overleftarrow{p}-q p\right)+(\overleftarrow{q} p-\vec{q} \vec{p})=0 \\
& -(\ln q)_{x y}+(\overleftarrow{q} \bar{p}-\vec{q} \vec{p})_{y}+(\overleftarrow{q} p-\vec{q} \vec{p})_{x}+\widehat{K}(q+\vec{q} q \vec{p} \vec{p}+q \overleftarrow{q} p \bar{p})=0 \\
& (\ln \bar{p})_{x}+\left(\overleftarrow{q}^{2} \overleftarrow{p}-q \bar{p}\right)+(\overleftarrow{q} \bar{p}-\vec{q} \vec{p})=0
\end{aligned}
$$

где $\widehat{K} \theta=\overleftarrow{\theta}-2 \theta+\vec{\theta}$.

В случае $p=\bar{p}=0$ мы возврашаемся к обычной цепочке Тоды $(У Т(1,1))$. Случай $\bar{p}=0, p \neq 0$ (или аналогичный ему $p=0, \bar{p} \neq 0$ ) соответствует цепочке УТ $(1,2)$ с уравнениями

$$
\begin{gathered}
-(\ln q)_{x y}+(\overleftarrow{q} p-\vec{q} \vec{p})_{x}+\overleftarrow{q}-2 q+\vec{q}=0 \\
(\ln p)_{y}+(\overleftarrow{q} 2 \overleftarrow{p}-q p)+(\overleftarrow{q} p-\vec{q} \vec{p})=0
\end{gathered}
$$

Последняя система представляет самостоятельный интерес, однако всегда может рассматриваться как редукция цепочки УТ $(2,2)$ при соответствуюшем выборе произвольных функций, определяющих ее общее решение.

Ниже мы приведем обшее решение системы (9), переписав ее предварительно в более обозримом виде.

Воспользуемся следуюшими подстановками:

$$
p=\exp (\overleftarrow{s}+s), \quad \bar{p}=\exp (\overleftarrow{t}+t), \quad \theta=q \exp (s+t)
$$

В новых переменных (9) принимает форму

$$
\begin{aligned}
& (\exp (-s))_{y}=\theta \overleftarrow{\exp }(-t)-\theta \overrightarrow{\exp }(-t), \quad(\exp (-t))_{x}=\theta \overleftarrow{\exp }(-s)-\theta \overrightarrow{\exp }(-s) \\
& -(\ln \theta)_{x y}+\widehat{K}(\theta \exp (-(s+t))+\theta \overleftarrow{\theta}+\theta \vec{\theta})=0
\end{aligned}
$$

или после окончательной идентификации $p^{(1}=\exp (-s), \bar{p}^{(1}=\exp (-t)$

$$
\begin{aligned}
& \left(p^{(1}\right)_{y}=\bar{p}^{\overleftarrow{C 1}} \theta-\bar{p}^{\overrightarrow{(1}^{1}} \theta, \quad\left(\bar{p}^{(1}\right)_{x}=p^{\overleftarrow{{ }_{1}}} \theta-p^{\overrightarrow{(1}^{1}} \theta \\
& -(\ln \theta)_{x y}+\widehat{K}\left(p^{(1} \bar{p}^{(1} \theta+\theta \overleftarrow{\theta}+\theta \vec{\theta}\right)=0 .
\end{aligned}
$$

Формы (9) и (11) описания цепочки УТ $(2,2)$ по сути абсолютно эквивалентны друг другу. Последняя более привлекательна чисто визуальной простотой. 
Для того чтобы записать обшее решение (9) или (11), нам потребуются некоторые результаты из [8], которые мы приведем в конспективном виде, применительно к данному случаю. В основе всей конструкции лежат два уравнения $S$-матричного типа:

$$
\begin{aligned}
& \left(M_{+}\right)_{y}=M_{+} L_{+} \equiv M_{+}\left(\sum_{1}^{r} Y_{\alpha}^{+1} \bar{\phi}_{\alpha}^{1}+\sum_{1}^{r-1} Y_{\beta}^{+2} \bar{\phi}_{\beta}^{2}\right), \\
& \left(M_{-}\right)_{x}=M_{-} L_{-} \equiv M_{-}\left(\sum_{1}^{r} X_{\alpha}^{-} \phi_{\alpha}^{1}+\sum_{1}^{r-1} Y_{\beta}^{-2} \phi_{\beta}^{2}\right),
\end{aligned}
$$

где $r$ - ранг полупростой алгебры, $Y^{ \pm 2}$ определены в $(6), \phi^{1,2}=\phi^{1,2}(x), \bar{\phi}^{1,2}=\bar{\phi}^{1,2}(y)$.

"Лагранжианы" $L^{ \pm}$уравнения (12) - соответственно верхняя и нижняя треугольные матрицы. По этой причине решения уравнений (12) могут быть записаны в явном виде в квадратурах.

Решение исходной задачи может быть представлено в терминах матричных элементов единственного элемента группы $S L(n+1, R)$ (с алгеброй $A_{n}$ )

$$
K=\exp (h \Phi) M_{-}^{-1} M_{+} \exp (-h \bar{\Phi}) \equiv m_{-}^{-1} m_{+} .
$$

Как вытекает из их определения, элементы группы $m_{ \pm}$удовлетворяют уравнениям

$$
\begin{aligned}
\left(m_{+}\right)_{y} & =m_{+}\left(-(h \bar{\Phi})_{y}+\sum Y_{j}^{+1}\left(\bar{\nu}_{j+1}-\bar{\nu}_{j-1}\right)+\sum Y_{j}^{+2}\right) \equiv \\
& \equiv m_{+}\left(\exp (h \bar{\Phi}) L_{+} \exp (-h \bar{\Phi})-(h \bar{\Phi})_{y}\right), \\
\left(m_{-}\right)_{x} & =m_{-}\left((h \Phi)_{x}+\sum Y_{j}^{-1}\left(\nu_{j+1}-\nu_{j-1}\right)+\sum Y_{j}^{-2}\right) \equiv \\
& \equiv m_{-}\left(\exp (h \Phi) L_{-} \exp (-h \Phi)-(h \Phi)_{x}\right) .
\end{aligned}
$$

Последние равенства определяют все дополнительно введенные функции и соотношения меж ду ними.

Через $\| i\rangle,(\langle i \|)$ обозначим минимальный вектор $i$-го фундаментального представления алгебры $A_{n}$ со следуюшими свойствами:

$$
\begin{aligned}
& \left.\left.X_{\alpha}^{-} \| i\right\rangle=0, \quad h_{s} \| i\right\rangle=-\delta_{s, i}, \quad\left\langle i \| X_{\alpha}^{+}=0, \quad\left\langle i \| h_{s}=-\delta_{s, i},\right.\right. \\
& \left.\left.X_{\alpha}^{+} \| i\right\rangle=\delta_{\alpha, i} X_{i}^{+} \| i\right\rangle, \quad\left\langle i \| X_{\alpha}^{-}=\delta_{\alpha, i}\left\langle i \| X_{i}^{-} .\right.\right.
\end{aligned}
$$

В дальнейшем мы используем следуюшие сокрашения:

$$
\begin{array}{ll}
{[i]=\langle i\|K\| i\rangle,} & \theta_{i}=\frac{[i+1][i-1]}{[i]^{2}}, \\
\alpha_{i j \ldots l} \equiv \frac{\left\langle i\left\|X_{i}^{-} X_{j}^{-} \ldots X_{l}^{-} K\right\| i\right\rangle}{\langle i\|K\| i\rangle}, & \bar{\alpha}_{i j \ldots l} \equiv \frac{\left\langle i\left\|K X_{l}^{+} \ldots X_{j}^{+} X_{i}^{+}\right\| i\right\rangle}{\langle i\|K\| i\rangle} .
\end{array}
$$


В этих обозначениях обшее решение системы (11) вьглядит так:

$$
\begin{aligned}
& p_{i}^{(1}=\bar{\nu}_{i+1}-\bar{\alpha}_{i+1}-\bar{\nu}_{i-1}+\bar{\alpha}_{i-1} \equiv\left(\theta_{i}\right)^{-1}\left(\alpha_{i}\right)_{y}, \\
& \theta_{i}=\frac{[i+1][i-1]}{[i]^{2}}, \\
& \bar{p}_{i}^{(1}=\nu_{i+1}-\alpha_{i+1}-\nu_{i-1}+\alpha_{i-1} \equiv\left(\theta_{i}\right)^{-1}\left(\bar{\alpha}_{i}\right)_{x} .
\end{aligned}
$$

Общее решение (9) в терминах (16) можно представить в виде

$$
q_{i}=p_{i}^{(1} \bar{p}_{i}^{(1} \theta_{i}, \quad \bar{p}_{i}=\frac{1}{\bar{p}_{i}^{(1} \bar{p}_{i+1}^{(1}}, \quad p_{i}=\frac{1}{p_{i}^{(1} p_{i+1}^{(1}} .
$$

Проверка справедливости сделанных выше утверждений проводится с помощью базового тождества между матричными элементами последовательных фундаментальных представлений:

$$
\operatorname{Det}_{2}\left(\begin{array}{cc}
\langle i\|K\| i\rangle & \left\langle i\left\|X_{i}^{-} K\right\| i\right\rangle \\
\left\langle i\left\|K X_{i}^{+}\right\| i\right\rangle & \left\langle i\left\|X_{i}^{-} K X_{i}^{+}\right\| i\right\rangle
\end{array}\right)=[i+1][i-1]
$$

На самом деле (17) есть не что иное, как знаменитое тождество Якоби, связываюшее детерминанты $(i, i+1, i-1)$-х порядков и записанное более экономным способом [5].

Доказательство (17) читатель может найти в [9] (см. также дополнение). Все другие необходимые соотношения являются прямыми следствиями (17).

Используя приведенные формулы, нетрудно проверить равенство

$$
(\ln [i])_{x y}=\theta_{i} \theta_{i+1}+\theta_{i} \theta_{i-1}+\bar{p}_{i}^{(1} p_{i}^{(1} \theta_{i}
$$

а также ряд других соотношений подобного рода (в частности, тождеств (16)). С деталями вычислений читатель может познакомиться в пятом разделе.

Общее решение для цепочки УТ $(1,2)$ содержится среди приведенных выше. С помощью преобразований такого же типа, как переход от (9) к (11), уравнения (10) переписываются в эквивалентной форме:

$$
\phi_{y}=\overleftarrow{\theta}-\vec{\theta}, \quad(\ln \theta)_{x y}=\overleftarrow{\phi} \theta-2 \phi \theta+\vec{\phi} \theta
$$

Общее решение (18) таково: $\phi$ совпадает с $p_{i}^{(1}$ из (16), а в выражении для $\theta$ требуется небольшая модификация:

$$
\theta_{i}=\nu_{i} \bar{\phi}_{i}^{1} \frac{\langle i-1\|K\| i-1\rangle\langle i+1\|K\| i+1\rangle}{(\langle i\|K\| i\rangle)^{2}} .
$$

В уравнении (12), определяющем $M_{ \pm}$, необходимо положить $\bar{\phi}_{i}^{2}=0$.

Явный вид (16) показывает, что решение полностью определяется единственным элементом группы $K$, а потому естественно предположить, что задачи, связанные с УТ-цепочками, закодированы в его матричных элементах. 


\section{4. ПАРАМЕТРЫ ЭВОЛЮЦИИ - ГАМИЛЬТОНОВЫ ПОТОКИ}

В этом разделе вводятся эволюционные параметры и предлагается методпостроения систем уравнений, инвариантных относительно УТ-подстановок. Обсуждение начинается с обычной УТ $(1,1)$-цепочки, для которой решение было найдено ранее другими методами [3].

4.1. Двумерная иерархия Деви-Стюартсона. В этом случае элемент группы $m_{+}$удовлетворяет уравнению

$$
m_{+}^{\prime} \equiv\left(m_{+}\right)_{y}=m_{+}\left(-(h \bar{\Phi})^{\prime}+\sum Y_{j}^{+1}\right)
$$

и определяется набором произвольных функций $\bar{\Phi}_{i}(y)$. Допустим, что помимо $y$ последние функции зависят также от переменной $t_{2}$ таким образом, чтобы уравнение

$$
\dot{m}_{+} \equiv\left(m_{+}\right)_{t_{2}}=m_{+}\left(-(h \bar{\Phi})^{\cdot}+\sum Y_{j}^{+1} \mu_{j}-\sum Y_{j}^{+2}\right)
$$

было совместно с (19). Разрешая тождества Маурера-Картана, приходим к системе соотношений

$$
\mu_{i}=-\bar{\Phi}_{i+1}+\bar{\Phi}_{i-1}, \quad \mu_{i}^{\prime}+(k \bar{\Phi})_{i}-\mu_{i}(k \bar{\Phi})^{\prime}=0,
$$

где, как обычно, $(k f)_{i}=-f_{i+1}+2 f_{i}-f_{i-1}$. Итак, $(21)$ эквивалентна системе уравнений

$$
\dot{\bar{\Phi}}_{i}-\dot{\bar{\Phi}}_{i-1}+\bar{\Phi}_{i}^{\prime \prime}+\bar{\Phi}_{i-1}^{\prime \prime}+\left(\bar{\Phi}_{i}^{\prime}-\bar{\Phi}_{i-1}^{\prime}\right)^{2}=0
$$

(во всех случаях из условия $b_{i}=b_{i+1}$ мы заключали, что $b_{i}=0$ ).

Решение для цепочки (22) можно представить в терминах $N$ линейно независимых решений одномерного уравнения Шредингера $(N-$ число узлов цепочки $(22))$

$$
\dot{\Psi}=\Psi^{\prime \prime}+V\left(t_{2}, y\right) \Psi,
$$

где $V$ - произвольная функция пространственной $y$ и временной $t_{2}$ координат [3].

Цепочка (22) индуцирует систему Деви-Стюартсона [10]. Чтобы это показать, вычислим пространственную и временную производные матричного элемента $[i]$ (мы используем обозначения (15) и последуюшие). Как следствие (19), (20) и (22) имеем

$$
\begin{aligned}
& (\ln [i])^{\prime}=\dot{\bar{\Phi}}_{i}+\mu_{i} \bar{\alpha}_{i}-\bar{\alpha}_{i, i+1}+\bar{\alpha}_{i, i-1}, \\
& (\ln [i])^{\prime}=\left(\bar{\Phi}_{i}\right)^{\prime}+\bar{\alpha}_{i}, \\
& \frac{[i]^{\prime \prime}}{[i]}=\left(\bar{\Phi}_{i}\right)^{\prime \prime}+\left(\bar{\Phi}_{i}^{\prime}\right)^{2}+\left(\bar{\Phi}_{i+1}+\bar{\Phi}_{i-1}\right)^{\prime} \bar{\alpha}_{i}+\bar{\alpha}_{i, i+1}+\bar{\alpha}_{i, i-1} .
\end{aligned}
$$

Исключая $\dot{\bar{\Phi}}_{i}, \bar{\Phi}_{i}^{\prime}, \bar{\Phi}_{i}^{\prime \prime}$ из $(22)$ с помошью $(23)$, приходим к следуюшему ключевому равенству:

$$
\left(\ln \frac{[i]}{[i-1]}\right)+(\ln ([i][i-1]))^{\prime \prime}+\left(\left(\ln \frac{[i]}{[i-1]}\right)^{\prime}\right)^{2}=2\left(\bar{\alpha}_{i-1, i}+\bar{\alpha}_{i, i-1}-\bar{\alpha}_{i-1} \bar{\alpha}_{i}\right),
$$


примечательному тем, что его правая часть тождественно равна нулю. Доказательство этого факта приведено в дополнении.

Вводя в рассмотрение функции

$$
v=\frac{[i]}{[i-1]}, \quad u=\frac{[i-2]}{[i-1]}
$$

имея в виду тождества (эквивалентные уравнениям цепочки Тоды)

$$
(\ln [i])_{x y}=\frac{[i+1][i-1]}{[i]^{2}}
$$

и справедливость (24) для произвольных $i$, мы заключаем, что функции $u, v$ удовлетворяют следующей системе уравнений:

$$
-\dot{u}+u_{y y}+2 u \int d x(u v)_{y}=0, \quad \dot{v}+v_{y y}+2 v \int d x(u v)_{y}=0 .
$$

Это в точности система Деви-Стюартсона [10], в одномерном случае $\left(\partial_{x}=\partial_{y}\right)$ переходящая в нелинейное одномерное уравнение Шредингера.

В общем случае дополнительное уравнение (20), определяющее зависимость произвольных функций от параметра эволюции, следует заменить условием разложимости алгеброзначной функции $m_{+}^{-1} \dot{m}_{+}$по генераторам алгебры с градуировочными индексами, не превышающими заданное натуральное число $d_{1}$. Возникаюшая система уравнений определяет зависимость функций $\bar{\Phi}_{i}$ от параметра эволюции $\bar{t}_{r}$ и пространственной координаты $y$. Как следствие возникает $d_{1}$-е уравнение иерархии Деви-Стюартсона. Другими методами эта задача была решена в явном виде в [3, 11].

В одномерном случае описанная выше конструкция эквивалентна многовременному формализму и технике гамильтоновых потоков [4].

Очевидно, что все проделанное выше можно повторить по отношению к пространственной координате $x$ в паре с элементом $m_{-}$.

В итоге мы получаем наборы правых $\left(\bar{t}_{d}\right)$ и левых $\left(t_{d}\right)$ параметров эволюции, соответствуюшие системы уравнений, инвариантные относительно подстановки УТ $(1,1)$, и набор их солитоноподобных решений.

4.2. Случай УТ(2.2). Воспользуемся техникой предыдущего раздела для построения интегрируемой системы уравнений, инвариантной относительно подстановки (11), т.е. относительно преобразований УТ $(2,2)$. Соответствуюшие вычисления хотя и несложны по своей сути, имеют весьма громоздкий вид. Поэтому мы опускаем, как правило, их подробное описание, приводя лишь окончательные результаты. Все необходимые для их проверки формулы содержатся в пятом разделе и в дополнении. Возможно, использование более удачных обозначений могло бы значительно упростить процедуру вычислений. 
В случае УТ $(2,2)$ элемент $m_{+}$удовлетворяет уравнению (см. раздел 3 )

$$
\begin{aligned}
\left(m_{+}\right)^{\prime} & =m_{+}\left(-(h \bar{\Phi})^{\prime}+\sum Y_{j}^{+1} \bar{\phi}_{j}+\sum Y_{j}^{+2}\right) \equiv \\
& \equiv m_{+}\left(\exp (h \bar{\Phi}) L_{+} \exp (-h \bar{\Phi})-(h \bar{\Phi})^{\prime}\right), \quad f^{\prime} \equiv f_{y}, \quad \bar{\phi}_{j}=\left(\bar{\nu}_{j+1}-\bar{\nu}_{j-1}\right) .
\end{aligned}
$$

Соответствуюший оператор $t_{2}$ дифференширования имеет вид

$$
\dot{m}_{+}=m_{+}\left(-(h \dot{\bar{\Phi}})+\sum Y_{j}^{+1} \mu_{j}^{(1}+\sum Y_{j}^{+2} \mu_{j}^{(2}+\sum Y_{j}^{+3} \mu_{j}^{(3}-\sum Y_{j}^{+4}\right) .
$$

Условие самосогласованности (тождества Маурера-Картана) позволяет выразить все функции $\mu_{i}^{(s}$ из $(26)$ в терминах $\bar{\Phi}_{i}, \bar{\nu}_{i}$ и получить систему (цепочку) уравнений, которой последние как функции аргументов $y, t_{2}$ удовлетворяют.

Используя коммутационные соотношения (7), приходим к искомой функциональной зависимости:

$$
\begin{aligned}
& \mu_{i}^{(3}=\bar{\phi}_{i+2}+\bar{\phi}_{i}=\bar{\nu}_{i+3}-\bar{\nu}_{i-1}, \quad \mu_{i}^{(2}=\bar{\Phi}_{i+1}^{\prime}-\bar{\Phi}_{i+2}^{\prime}-\bar{\Phi}_{i}^{\prime}+\bar{\Phi}_{i-1}^{\prime}+\bar{\phi}_{i} \bar{\phi}_{i+1}, \\
& \mu_{i}^{(1}=-\left(\bar{\nu}_{i+1}-\bar{\nu}_{i-1}\right)\left(\bar{\Phi}_{i+1}^{\prime}-\bar{\Phi}_{i-1}^{\prime}\right)-\left(\bar{\nu}_{i+1}^{\prime}+\bar{\nu}_{i-1}^{\prime}\right),
\end{aligned}
$$

и системе зацепляющихся уравнений, которым функции $\bar{\Phi}, \bar{\nu}$ удовлетворяют (ср. с (22)):

$$
\begin{aligned}
& \dot{\bar{\Phi}}_{i+1}-\dot{\bar{\Phi}}_{i-1}+\bar{\Phi}_{i+1}^{\prime \prime}+\bar{\Phi}_{i-1}^{\prime \prime}+\left(\bar{\Phi}_{i}^{\prime}-\bar{\Phi}_{i+1}^{\prime}\right)^{2}+\left(\bar{\Phi}_{i}^{\prime}-\bar{\Phi}_{i-1}^{\prime}\right)^{2}=2 \bar{\nu}_{i}^{\prime}\left(\bar{\nu}_{i+1}-\bar{\nu}_{i-1}\right) \\
& \left(\bar{\nu}_{i+1}-\bar{\nu}_{i-1}\right)+\left(\bar{\nu}_{i+1}+\bar{\nu}_{i-1}\right)^{\prime \prime}-2 \bar{\nu}_{i+1}^{\prime}\left(\bar{\Phi}_{i}^{\prime}-\bar{\Phi}_{i-1}^{\prime}\right)-2 \bar{\nu}_{i-1}^{\prime}\left(\bar{\Phi}_{i}^{\prime}-\bar{\Phi}_{i+1}^{\prime}\right)+ \\
& \quad+\left(\bar{\nu}_{i+1}-\bar{\nu}_{i-1}\right)\left[\left(\dot{\bar{\Phi}}_{i+1}-2 \dot{\bar{\Phi}}_{i}\right)+\dot{\bar{\Phi}}_{i-1}+\bar{\Phi}_{i+1}^{\prime \prime}-\bar{\Phi}_{i-1}^{\prime \prime}+\right. \\
& \left.\quad+\left(\bar{\Phi}_{i+1}^{\prime}\right)^{2}-\left(\bar{\Phi}_{i-1}^{\prime}\right)^{2}-2\left(\bar{\Phi}_{i+1}^{\prime}-\bar{\Phi}_{i-1}^{\prime}\right) \bar{\Phi}_{i}^{\prime}\right]=0
\end{aligned}
$$

Ниже мы несколько отступим от принятых ранее обозначений и будем рассматривать УТ $(2,2)$ в переменных

$$
p_{i}=\alpha_{i}-\nu_{i}, \quad \bar{p}_{i}=\bar{\alpha}_{i}-\bar{\nu}_{i} .
$$

Тогда, как это вытекает из (16), УТ(2, 2)-подстановка принимает вид

$$
\begin{aligned}
& \left(p_{i}\right)_{y}=\theta_{i}\left(\bar{p}_{i-1}-\bar{p}_{i+1}\right), \quad\left(\bar{p}_{i}\right)_{x}=\theta_{i}\left(p_{i-1}-p_{i+1}\right) \\
& (\ln \theta)_{x y}=\widehat{K}\left(p_{y} \bar{p}_{x} \theta+\theta \overleftarrow{\theta}+\theta \vec{\theta}\right)
\end{aligned}
$$

Вводим функции

$$
v_{i}=\frac{[i+1]}{[i]}, \quad u_{i}=\frac{[i-1]}{[i]} .
$$

Очевидно, $\theta_{i} \equiv u_{i} v_{i}, u_{i+1}=v_{i}^{-1}$. 
Следуюшие соотношения - прямое следствие введенных выше определений (что может быть проверено прямым расчетом с помошью соответствуюших формул пятого раздела и дополнения):

$$
\begin{aligned}
& \frac{\dot{v}_{i}}{v_{i}}+\frac{v_{i}^{\prime \prime}}{v_{i}}+2\left(\bar{\alpha}_{i, i-1}-\bar{\nu}_{i-1} \bar{\alpha}_{i}\right)^{\prime}-2 \bar{p}_{i}^{\prime} \bar{p}_{i+1}=V_{i}\left(y, t_{2}\right), \\
& -\frac{\dot{u}_{i}}{u_{i}}+\frac{u_{i}^{\prime \prime}}{u_{i}}-2\left(\bar{\alpha}_{i, i+1}-\bar{\nu}_{i+1} \bar{\alpha}_{i}\right)^{\prime}+2 \bar{p}_{i}^{\prime} \bar{p}_{i-1}=U_{i}\left(y, t_{2}\right), \\
& \dot{p}_{i}=-p_{i}^{\prime \prime}-2\left(\ln v_{i-1}\right)^{\prime}+2 \theta_{i} \bar{p}_{i-1}^{\prime} \\
& V_{i}=\dot{\bar{\Phi}}_{i+1}-\dot{\bar{\Phi}}_{i}+\bar{\Phi}_{i+1}^{\prime \prime}-\bar{\Phi}_{i}^{\prime \prime}+\left(\bar{\Phi}_{i}^{\prime}-\bar{\Phi}_{i+1}^{\prime}\right)^{2}-2 \bar{\nu}_{i}^{\prime} \bar{\nu}_{i+1}, \\
& U_{i}=-\dot{\bar{\Phi}}_{i-1}+\dot{\bar{\Phi}}_{i}+\bar{\Phi}_{i-1}^{\prime \prime}-\bar{\Phi}_{i}^{\prime \prime}+\left(\bar{\Phi}_{i}^{\prime}-\bar{\Phi}_{i-1}^{\prime}\right)^{2}+2 \bar{\nu}_{i}^{\prime} \bar{\nu}_{i-1} .
\end{aligned}
$$

Для того чтобы иметь замкнутую систему, необходимо прежде всего выразить функции $\bar{\alpha}_{i \pm 1}, \bar{\alpha}_{i, i \pm 1}$ через функции, участвуюшие в вышеприведенных равенствах. Это достигается с помощью тождества

$$
\left(\bar{\alpha}_{i, i \pm 1}-\bar{\nu}_{i \pm 1} \bar{\alpha}_{i}\right)_{x}=\mp \theta_{i} \theta_{i \pm 1}+\theta_{i} \bar{p}_{i \pm 1}\left(\bar{p}_{i}\right)_{x}
$$

которое нетрудно проверить. Теперь вышеприведенную систему, учитывая уравнения подстановки (28), можно переписать в виде системы из восьми уравнений для восьми неизвестных функций $v_{i+1}, v_{i}, u_{i}, u_{i-1}, p_{i}, p_{i+1}, \bar{p}_{i}, \bar{p}_{i-1}$ :

$$
\begin{aligned}
& \dot{v}_{i+1}+v_{i+1}^{\prime \prime}+2 v_{i} \bar{p}_{i+1}^{\prime} p_{i+1}^{\prime}+2 v_{i+1} \bar{p}_{i+1} \bar{p}_{i}^{\prime}-2 v_{i+1} \int d x\left[-u_{i} v_{i+1}+\bar{p}_{i+1}\left(\bar{p}_{i}\right)_{x}\right]^{\prime}=0, \\
& \dot{v}_{i}+v_{i}^{\prime \prime}-2 v_{i} \bar{p}_{i+1} \bar{p}_{i}^{\prime}+2 v_{i} \int d x\left[v_{i} u_{i-1}+\bar{p}_{i-1}\left(\bar{p}_{i}\right)_{x}\right]^{\prime}=0, \\
& -\dot{u}_{i}+u_{i}^{\prime \prime}+2 u_{i} \bar{p}_{i-1} \bar{p}_{i}^{\prime}-2 u_{i} \int d x\left[-u_{i} v_{i+1}+\bar{p}_{i+1}\left(\bar{p}_{i}\right)_{x}\right]^{\prime}=0, \\
& -\dot{u}_{i-1}+u_{i-1}^{\prime \prime}+2 u_{i} \bar{p}_{i-1}^{\prime} p_{i-1}^{\prime}-2 u_{i-1} \bar{p}_{i-1} \bar{p}_{i}^{\prime}+2 u_{i-1} \int d x\left[v_{i} u_{i-1}+\bar{p}_{i-1}\left(\bar{p}_{i}\right)_{x}\right]^{\prime}=0, \\
& \dot{p}_{i}+p_{i}^{\prime \prime}-2\left(\ln u_{i}\right)^{\prime} p_{i}^{\prime}-2 u_{i} v_{i} \bar{p}_{i-1}^{\prime}=0, \\
& \dot{p}_{i-1}+p_{i-1}^{\prime \prime}-2\left(\ln u_{i}\right)^{\prime} p_{i-1}^{\prime}-2 \frac{u_{i-1}}{u_{i}} \bar{p}_{i}^{\prime}=0, \\
& \left(\theta_{i}^{-1}\left(\bar{p}_{i}\right)_{x}\right)^{\cdot}=p_{i+1}^{\prime \prime}+p_{i-1}^{\prime \prime}+2 p_{i+1}^{\prime}\left(\ln v_{i}\right)^{\prime}+2 p_{i-1}^{\prime}\left(\ln u_{i}\right)^{\prime}-2 \bar{p}_{i}^{\prime}\left(\theta_{i+1}-\theta_{i-1}\right), \\
& \left(\theta_{i-1}^{-1}\left(\bar{p}_{i-1}\right)_{x}\right)^{\cdot}=p_{i}^{\prime \prime}+p_{i-2}^{\prime \prime}+2 p_{i}^{\prime}\left(\ln v_{i-1}\right)^{\prime}+2 p_{i-2}^{\prime}\left(\ln u_{i-1}\right)^{\prime}-2 \bar{p}_{i-1}^{\prime}\left(\theta_{i}-\theta_{i-2}\right) .
\end{aligned}
$$

Из (29) непосредственно видно, что 8 функций

$$
\begin{array}{llll}
p_{i}=\alpha_{i}-\nu_{i}, & p_{i-1}=\alpha_{i-1}-\nu_{i-1}, & \bar{p}_{i}=\bar{\alpha}_{i}-\bar{\nu}_{i}, & \bar{p}_{i-1}=\bar{\alpha}_{i-1}-\bar{\nu}_{i-1}, \\
v_{i}=\frac{[i+1]}{[i]}, & v_{i+1}=\frac{[i+2]}{[i+1]}, & u_{i}=\frac{[i-1]}{[i]}, & u_{i-1}=\frac{[i-2]}{[i-1]}
\end{array}
$$

при произвольном выборе $i$ являются частными решениями одной и той же системы (получающейся, если искомые функции в (29) представить в безындексной форме). 
Теперь мы можем пояснить ситуацию с решением системы (27). Очевидно, что система (29) обладает частным решением вида

$$
u_{0}=u_{-1}=p_{0}=\bar{p}_{0}=p_{-1}=\bar{p}_{-1}=0 .
$$

Действительно, в случае алгебры $A_{n}$ (при произвольном $n$ ) все перечисленные матричные элементы отсутствуют. Для остающихся неизвестных функций $v_{0}, v_{1}, p_{1}, \bar{p}_{1}$ как следствие (29) возникает замкнутая система уравнений

$$
\begin{aligned}
\dot{v}_{0}+v_{0}^{\prime \prime} & =V_{0} v_{0}, & \left(p_{1} v_{0}\right)^{\cdot}+\left(p_{1} v_{0}\right)^{\prime \prime} & =V_{0}\left(p_{1} v_{0}\right), \\
\left(\bar{p}_{1} v_{0}\right)^{\cdot}+\left(\bar{p}_{1} v_{0}\right)^{\prime \prime} & =U_{0}\left(\bar{p}_{1} v_{0}\right), & \dot{v}_{1}+v_{1}^{\prime \prime}-U_{0} v_{1} & =-2 p_{1}^{\prime} \bar{p}_{1}^{\prime} v_{0}
\end{aligned}
$$

(появление произвольных функций $U_{0}, V_{0}$ связано с неоднозначностью неопределенного интеграла $\left.\int d x 0=F\left(y, t_{2}\right)\right)$.

Мы видим, что функции $v_{0}, p_{1} v_{0}$ являются решениями одномерного однородного уравнения Шредингера с произвольной потенциальной функцией $V_{0}$. При этом $v_{1}$ и $\bar{p}_{1} v_{0}$ являются решениями (не)однородного уравнения Шредингера с потенциалом $U_{0}$ и известным источником $-2 p_{1}^{\prime} \bar{p}_{1}^{\prime} v_{0}$. В терминах различных решений указанной пары уравнений Шредингера можно представить обшее решение цепочки (27). К этой проблеме мы надеемся вернуться в другом месте.

В заключение настоящего раздела приведем $(1+1)$-интегрируемую систему, которая возникает в результате редукции $(29)$ на пространство одного переменного $(\partial / \partial x=$ $\partial / \partial y):$

$$
\begin{aligned}
& \dot{v}_{i+1}+v_{i+1}^{\prime \prime}+2 v_{i} \bar{p}_{i+1}^{\prime} p_{i+1}^{\prime}+2 v_{i+1}^{2} u_{i}=0, \\
& \dot{v}_{i}+v_{i}^{\prime \prime}+2 \frac{1}{u_{i}} \bar{p}_{i}^{\prime} p_{i}^{\prime}+2 v_{i}^{2} u_{i-1}=0 \\
& -\dot{u}_{i}+u_{i}^{\prime \prime}+2 \frac{1}{v_{i}} p_{i} \bar{p}_{i}^{\prime}+2 u_{i}^{2} v_{i+1}=0 \\
& -\dot{u}_{i-1}+u_{i-1}^{\prime \prime}+2 u_{i} \bar{p}_{i-1}^{\prime} p_{i-1}^{\prime}+2 u_{i-1}^{2} v_{i}=0 \\
& \dot{p}_{i}+p_{i}^{\prime \prime}-2\left(\ln u_{i}\right)^{\prime} p_{i}^{\prime}-2 u_{i} v_{i} \bar{p}_{i-1}^{\prime}=0 \\
& \dot{p}_{i-1}-p_{i-1}^{\prime \prime}-2\left(\ln u_{i}\right)^{\prime} p_{i-1}^{\prime}-2 \frac{u_{i-1}}{u_{i}} \bar{p}_{i}^{\prime}=0, \\
& \dot{\bar{p}}_{i}+\bar{p}_{i}^{\prime \prime}-2\left(\ln u_{i}\right)^{\prime} \bar{p}_{i}^{\prime}-2 u_{i} v_{i} p_{i-1}^{\prime}=0 \\
& \dot{\bar{p}}_{i-1}-\bar{p}_{i-1}^{\prime \prime}-2\left(\ln u_{i}\right)^{\prime} \bar{p}_{i-1}^{\prime}-2 \frac{u_{i-1}}{u_{i}} p_{i}^{\prime}=0 .
\end{aligned}
$$

Уравнения системы (30) инвариантны по отношению к преобразованиям одномерного варианта УТ $(2,2)$-подстановки $(28)$ и обладают бесконечным набором сохраняюшихся величин.

\section{5. УРАВНЕНИЯ УТ $\left(m_{1}, m_{2}\right)$-ЦЕПОЧКИ И ИХ ОБШЕЕ РЕШЕНИЕ}

В настояшем разделе собраны вспомогательные равенства и соотношения. С их помошью задача построения УТ $\left(m_{1}, m_{2}\right)$-цепочек уравнений и их обших решений переходит в область чисто технических манипуляций. 
Пусть пара групповых элементов $m^{ \pm}$удовлетворяет уравнениям, обобщающим (14):

$$
\begin{aligned}
& m_{y}^{+}=m^{+}\left(-(h \bar{\Phi})_{y}+\sum_{s=1}^{m_{1}}\left(Y^{s} \bar{\phi}^{(s)}\right) \equiv m^{+} L^{+}\right. \\
& \left(Y^{s} \bar{\phi}^{(s)} \equiv \sum_{i}\left(Y_{i}^{s} \bar{\phi}_{i}^{(s)}\right), \quad\left(Y^{s} \phi^{(s}\right)^{T} \equiv \sum_{i}\left(Y_{i}^{-s} \phi_{i}^{(s)}\right)\right. \\
& m_{x}^{-}=m^{-}\left((h \Phi)_{x}-\sum_{s=1}^{m_{2}}\left(Y^{s} \bar{\phi}^{(s}\right)^{T}\right) \equiv m^{-} L^{-}
\end{aligned}
$$

где генераторы $Y^{ \pm s}$ и их взаимные коммутаторы определены в (6) и ниже.

Наша ближайшая цель - установить правила дифференцирования матричных элементов в различных представлениях элемента группы

$$
k=m_{-}^{-1} m_{+} .
$$

Очевидно, что

$$
(\ln [i])_{x y}=[i]^{-2}\left(\begin{array}{cc}
\langle i\|k\| i\rangle & \left\langle i\left\|L^{-} k\right\| i\right\rangle \\
\left\langle i\left\|k L^{+}\right\| i\right\rangle & \left\langle i\left\|L^{-} k L^{+}\right\| i\right\rangle
\end{array}\right) .
$$

В соответствии с (15) при действии генераторов простых корней на минимальньй базисньй вектор отличны от нуля только $\left.X_{i}^{+} \| i\right\rangle \neq 0,\left\langle i \| X_{i}^{-} \neq 0\right.$. По этой причине действие операторов $L^{ \pm}$на минимальный вектор фундаментального представления может быть представлено в виде

$$
\left.\left.L^{+} \| i\right\rangle=l_{i}^{+} X_{i}^{+} \| i\right\rangle, \quad\left\langle i \| L^{-}=\left\langle i \| X_{i}^{-} l_{i}^{-},\right.\right.
$$

где $l_{i}^{ \pm}$- некоторые операторы, полиномиальные по генераторам положительных (отрицательных) простых корней. Например,

$$
\left.\left.\left(Y^{2} \bar{\phi}^{(2}\right) \| i\right\rangle=\left(\phi_{i-1}^{(2} X_{i-1}^{+}-\phi_{i}^{(2} X_{i+1}^{+}\right) X_{i}^{+} \| i\right\rangle,
$$

в этом случае $l_{i}^{+}=\phi_{i-1}^{(2} X_{i-1}^{+}-\phi_{i+1}^{(2} X_{i+1}^{+}$. Имея в виду этот факт и принимая во внимание (8), мы переписываем (33) в виде

$$
(\ln [i])_{x y}=[i]^{-2}\left(l_{i}^{-}\right)_{l}\left(l_{i}^{+}\right)_{r}[i-1][i+1],
$$

где теперь уже $\left(l_{i}^{-}\right)_{l}, \quad\left(l_{i}^{+}\right)_{r}-$ те же самые полиномы, построенные, однако, из генераторов простых корней, соответственно левого и правого присоединенного представлений. Нижеприведенные формулы иллюстрируют применение (34) к конкретному случаю $m_{1}=m_{2}=3$ в $(31)$ :

$$
\begin{aligned}
& \left(\alpha_{i}\right)_{y}=\theta_{i}\left(\bar{\phi}_{i}^{(1}+\bar{\phi}_{i-1}^{(2} \bar{\alpha}_{i-1}-\bar{\phi}_{i}^{(2} \bar{\alpha}_{i+1}+\bar{\alpha}_{i+1, i+2}-\bar{\alpha}_{i+1} \bar{\alpha}_{i-1}+\bar{\alpha}_{i-1, i-2}\right), \\
& \left(\alpha_{i, i+1}\right)_{y}=\alpha_{i+1}\left(\alpha_{i}\right)_{y}-\theta_{i} \theta_{i+1}\left(\bar{\phi}_{i}^{(2}+\bar{\alpha}_{i-1}-\bar{\alpha}_{i+2}\right) \\
& (\ln [i])_{x y}=\theta_{i+2} \theta_{i+1} \theta_{i}+\theta_{i+1} \theta_{i} \theta_{i-1}+\theta_{i-2} \theta_{i-1} \theta_{i}+ \\
& \quad+\theta_{i+1} \theta_{i}\left(\phi_{i}^{(2}+\alpha_{i-1}-\alpha_{i+2}\right)(. .)+\theta_{i-1} \theta_{i}\left(\phi_{i-1}^{(2}+\alpha_{i-2}-\alpha_{i+1}\right)(. .)+ \\
& \quad+\theta_{i}\left(\phi_{i}^{(1}+\phi_{i-1}^{(2} \alpha_{i-1}-\phi_{i}^{(2} \alpha_{i+1}+\alpha_{i+1, i+2}-\alpha_{i+1} \alpha_{i-1}+\alpha_{i-1, i-2}\right)(\ldots),
\end{aligned}
$$


где под (. . .) имеются в виду те же самые величины, что и в соответствуюших предшествующих сомножителях, с заменой всех функций $f$ на $\bar{f}$. Очевидно, что аналогичные (35) соотношения будут выполняться после замены $\alpha \rightarrow \bar{\alpha}, y \rightarrow x$ и наоборот.

Введем новые функции $p_{i}^{(1,2}, \bar{p}_{i}^{(1,2}$ :

$$
\begin{array}{ll}
p_{i}^{(1}=\theta_{i}^{1}\left(\bar{\alpha}_{i}\right)_{y}, & \bar{p}_{i}^{(1}=\theta_{i}^{1}\left(\alpha_{i}\right)_{x}, \\
p_{i}^{(2}=\phi_{i}^{(2}+\alpha_{i-1}-\alpha_{i+2}, & \bar{p}_{i}^{(2}=\bar{\phi}_{i}^{(2}+\bar{\alpha}_{i-1}-\bar{\alpha}_{i+2} .
\end{array}
$$

Используя еще раз (34), приходим к системе уравнений для функций $p_{i}^{(1,2}, \bar{p}_{i}^{(1,2}, \theta_{i}$ :

$$
\begin{gathered}
\left(p_{i}^{(2)}\right)_{y}=\theta_{i-1} \bar{p}_{i-1}^{(1}-\theta_{i+2} \bar{p}_{i+2}^{(1}, \quad\left(\bar{p}_{i}^{(2}\right)_{x}=\theta_{i-1} p_{i-1}^{(1}-\theta_{i+2} p_{i+2}^{(1}, \\
\left(p_{i}^{(1}\right)_{y}=\theta_{i-1} \bar{p}_{i-1}^{(1} p_{i-1}^{(2}-\theta_{i+1} \bar{p}_{i+1}^{(1} p_{i}^{(2}+\theta_{i-1} \theta_{i-2} \bar{p}_{i-2}^{(2}-\theta_{i+1} \theta_{i+2} \bar{p}_{i+1}^{(2}, \\
\left(\bar{p}_{i}^{(1}\right)_{x}=\theta_{i-1} p_{i-1}^{(1} \bar{p}_{i-1}^{(2}-\theta_{i+1} p_{i+1}^{(1} \bar{p}_{i}^{(2}+\theta_{i-1} \theta_{i-2} p_{i-2}^{(2}-\theta_{i+1} \theta_{i+2} p_{i+1}^{(2}, \\
\left(\ln \theta_{i}\right)_{x y}=\widehat{K}\left(\theta_{i} \theta_{i+1} \theta_{i+2}+\theta_{i} \theta_{i-1} \theta_{i-2}+\theta_{i} \theta_{i+1} p_{i}^{(2} \bar{p}_{i}^{(2}+\theta_{i-1} \theta_{i} p_{i-1}^{(2} \bar{p}_{i-1}^{(2}+\theta_{i} p_{i}^{(1} \bar{p}_{i}^{(1}\right) .
\end{gathered}
$$

Это как раз система уравнений УТ $(3,3)$-цепочки с известным обшим решением, которое определяется набором произвольных функций $\left(\Phi_{i}, \phi_{i}^{(1}, \phi_{i}^{(2}\right)$ и $\left(\bar{\Phi}_{i}, \bar{\phi}_{i}^{(1}, \bar{\phi}_{i}^{(2}\right)$ одного аргумента $(x, y)$, соответственно.

Буквально повторяя вычисления настоящего раздела или аналогичные из третьего раздела, приходим окончательно к уравнениям УТ $\left(m_{1}, m_{2}\right)$ интегрируемой подстановки:

$$
\begin{aligned}
& \left(p_{\alpha}^{(s)}\right)_{y}=\sum_{k=1}^{m_{1}-s}\left(p_{\alpha-k}^{(s+k} \bar{p}_{\alpha-k}^{(k} \prod_{i=1}^{k} \theta_{\alpha-k+i-1}-p_{\alpha}^{(s+k} \bar{p}_{\alpha+s}^{(k} \prod_{i=1}^{k} \theta_{\alpha+s+i-1}\right) \\
& \left(\ln \theta_{\alpha}\right)_{x y}=\widehat{K} \sum_{s=1}^{\min \left(m_{1}, m_{2}\right)} \sum_{k=0}^{s-1} p_{\alpha-k}^{(s} \bar{p}_{\alpha-k}^{(s} \prod_{i=1}^{s} \theta_{\alpha-s+i-1}, \quad p_{\alpha}^{\left(m_{1}\right.}=1, \quad \bar{p}_{\alpha}^{\left(m_{2}\right.}=1 \\
& \left(\bar{p}_{\alpha}^{(s)}\right)_{y}=\sum_{k=1}^{m_{2}-s}\left(\bar{p}_{\alpha-k}^{(s+k} p_{\alpha-k}^{(k} \prod_{i=1}^{k} \theta_{\alpha-k+i-1}-\bar{p}_{\alpha}^{(s+k} p_{\alpha+s}^{(k} \prod_{i=1}^{k} \theta_{\alpha+s+i-1}\right)
\end{aligned}
$$

\section{6. ЗАКЛЮЧИТЕЛЬНЫЕ ЗАМЕЧАНИЯ}

Обсудим сначала еше раз предлагаемую конструкцию, не вдаваясь в детали.

В основе построения лежат два групповых элемента $m_{ \pm}$, принадлежащие соответственно \pm разрешимым подгруппам (относительно некоторой градуировки) полупростой группы. Они определяются парой уравнений $S$-матричного типа:

$$
l_{+} \equiv m_{+}^{-1}\left(m_{+}\right)_{y}=\sum_{s=0}^{m_{1}} A^{+s}, \quad l_{-} \equiv m_{-}^{-1}\left(m_{-}\right)_{x}=\sum_{s=0}^{m_{2}} A^{-s} .
$$


Эти уравнения, чисто алгебраические по своей природе, представляют собой условие разложимости операторов $l_{ \pm}$по генераторам алгебры с градуировочными индексами, меньшими определенных заданных $m_{1}, m_{2}$.

Матричные элементы различных фундаментальных представлений элемента группы $K=m_{-}^{-1} m_{+}$удовлетворяют определенной системе тождеств, которые могут быть интерпретированы как уравнения точно интегрируемой цепочки УТ $\left(m_{1}, m_{2}\right)$.

На этом построение интегрируемого УТ $\left(m_{1}, m_{2}\right)$-отображения (подстановки) заканчивается.

Следующий шаг связан с введением параметров эволюции. На функции $\phi_{i}^{(s}(y), \phi_{i}^{(s}(x)$, до сего времени произвольные, накладываются дополнительные условия, которым должны удовлетворять элементы $m_{ \pm}$:

$$
m_{+}^{-1}\left(m_{+}\right)_{\bar{t}_{d_{1}}}=\sum_{s=0}^{d_{1}} B^{+s}, \quad m_{-}^{-1}\left(m_{-}\right)_{\bar{t}_{d_{2}}}=\sum_{s=0}^{d_{2}} B^{-s}
$$

Условия совместности (37) с (38) определяют явную зависимость функций $\bar{\phi}_{i}^{(s} \equiv$ $\bar{\phi}_{i}^{(s}\left(y, \bar{t}_{1}, \bar{t}_{2}, \ldots\right), \phi_{i}^{(s} \equiv \phi_{i}^{(s}\left(x, t_{1}, t_{2}, \ldots\right)$ как от эволюционных (временных) параметров, так и от пространственных координат $x, y$.

В результате возникают иерархии интегрируемых $(1+2)$ уравнений (каждая система иерархии определяется своим набором $d_{1}, d_{2}$ при фиксированных $\left.m_{1}, m_{2}\right)$. Все системы иерархии инвариантны относительно УТ $\left(m_{1}, m_{2}\right)$-подстановки.

В одномерном случае $(\partial / \partial y=\partial / \partial x)$ предлагаемая конструкция эквивалентна многовременному формализму или технике гамильтоновых потоков [4].

Теперь перечислим задачи, интересные с нашей точки зрения, которые могут быть решены в контексте результатов настояшей работы.

Не вызывает сомнения возможность прямого обобшения предложенной конструкции на случай суперсимметрии. На этом пути могут быть в явном виде построены не известные до сих пор интегрируемые иерархии в (2|2)-суперпространстве.

Исключительно интересна ситуация в квантовой области. Гайзенберговы операторы квантованной (в соответствии с каноническими правилами) двумерной цепочки Тоды [12] выражаются в терминах матричных элементов единственного оператора $k=$ $m_{-}^{-1} m_{+}$. Элементы $m_{ \pm}$являются решениями следуюших уравнений:

$$
\left(m_{+}\right)_{y}=m_{+}\left(\sum_{s=1}^{r} X_{s}^{+} \exp (k \bar{\phi}(y))\right)_{s}, \quad\left(m_{-}\right)_{x}=m_{-}\left(\sum_{s=1}^{r} X_{s}^{-} \exp (k \phi(x))\right)_{s}
$$

где теперь $X_{s}^{ \pm}$- генераторы простых корней уже квантовых алгебр, а $\phi(x)+\bar{\phi}(y)-$ квантованное поле двумерного уравнения Лапласа, $k$ - матрица Картана полупростой алгебры.

Сравнение (37) и (39) приводит к интригуюшему вопросу: с какими квантовыми алгебрами связаны уравнения квантованных УТ $\left(m_{1}, m_{2}\right)$-цепочек? 
В настояшей работе был рассмотрен единственный конкретный пример системы (п. 4.2), инвариантной относительно УТ $(2,2)$-подстановки. Однако мы надеемся и уверены, что сушествует универсальная процедура, позволяюшая в обшем случае УТ-подстановок находить решения уравнений симметрии столь же просто, как это было проделано в [3] в простейшем случае УТ $(1,1)$.

И, наконец, последнее замечание. Читатель, наверное, уже обратил внимание на значительное несоответствие между удивительной простотой и чисто алгебраической природой изначальных предположений (единственный групповой элемент $k$ и уравнения (37), (38), его определяющие), с одной стороны, и многочисленными рекуррентными соотношениями между матричными элементами различных фундаментальных представлений, для которых необходимы независимые обоснования, с другой. Наиболее вероятно, что последние рекуррентные соотношения являются прямыми следствиями уравнений $(37),(38)$ и групповых свойств элементов $m_{ \pm}$. Однако в настоящий момент автору неизвестно, как подобная информация может быть извлечена и возможно ли вообше это сделать. К этой увлекательной проблеме автор надеется вернуться в дальнейшем.

\section{ДОПОЛНЕНИЕ}

Рассмотрим функцию

$$
\begin{aligned}
R^{i}= & \left\langle i\left\|K X_{i-1}^{+} X_{i}^{+}\right\| i\right\rangle[i-1]+\left\langle i-1\left\|K X_{i}^{+} X_{i-1}^{+}\right\| i-1\right\rangle[i]- \\
& -\left\langle i\left\|K X_{i}^{+}\right\| i\right\rangle\left\langle i-1\left\|K X_{i-1}^{+}\right\| i-1\right\rangle,
\end{aligned}
$$

где $K$ - произвольный элемент группы $S L(n, R)$.

Из ее определения и свойств младшего вектора состояния $\| i\rangle(15)$ вытекает, что функция $R$ обрашается в нуль генераторами всех правых положительных и левых отрицательных простых корней. Это означает, что $R$ представима в виде линейной комбинации матричных элементов с обкладками из минимальных векторов.

Вычисление элементов Картана на $R$ приводит к результату

$$
h_{r}^{s} R^{i}=-\left(\delta_{s, i+1}+\delta_{s, i-2}\right) R^{i}, \quad h_{l}^{s} R^{i}=-\left(\delta_{s, i}+\delta_{s, i-1}\right) R^{i} .
$$

Отсюда вытекает, что функция $R^{i}$ справа и слева принадлежит к различным неприводимым представлениям. Последнее невозможно, и, следовательно, $R^{i}=0$. Так же может быть доказано тождество Якоби (см. третий раздел).

Ниже приведены простейшие базисные векторы $i$-го фундаментального представления. Они различаются числом повышаюших генераторов простых корней, примененных к минимальному вектору состояния.

Базисные векторы нулевого $\| i\rangle$ и первого $\left.X_{i}^{+} \| i\right\rangle$ порядков одномерны. Сушествуют два вектора второго порядка

$$
\left.\left.X_{i+1}^{+} X_{i}^{+} \| i\right\rangle, \quad X_{i-1}^{+} X_{i}^{+} \| i\right\rangle,
$$


три вектора третьего порядка

$$
\left.\left.\left.X_{i+2}^{+} X_{i+1}^{+} X_{i}^{+} \| i\right\rangle, \quad X_{i+1}^{+} X_{i-1}^{+} X_{i}^{+} \| i\right\rangle, \quad X_{i-2}^{+} X_{i-1}^{+} X_{i}^{+} \| i\right\rangle
$$

и, наконец, пять векторов четвертого порядка

$$
\begin{gathered}
\left.\left.\left.X_{i+3}^{+} X_{i+2}^{+} X_{i+1}^{+} X_{i}^{+} \| i\right\rangle, \quad X_{i+2}^{+} X_{i-1}^{+} X_{i+1}^{+} X_{i}^{+} \| i\right\rangle, \quad X_{i}^{+} X_{i+1}^{+} X_{i-1}^{+} X_{i}^{+} \| i\right\rangle \\
\left.\left.X_{i-2}^{+} X_{i-1}^{+} X_{i+1}^{+} X_{i}^{+} \| i\right\rangle, \quad X_{i-3}^{+} X_{i-2}^{+} X_{i-1}^{+} X_{i}^{+} \| i\right\rangle
\end{gathered}
$$

Все другие возможности приводят к векторам с нулевой нормой.

Благодарность. Автор благодарен Российскому фонду фундаментальных исследований (грант № 95-01-00249) за финансовую поддержку.

\section{Список литературы}

[1] D. B. Fairlie, A. N. Leznov. Phys. Lett. A. 1995. V. 199. P. 360-364.

[2] A. N. Leznov. Physica D. 1995. V. 87. P. 48-51; Discrete group of integrable mappings as a foundation of the theory of integrable systems. Preprint MPI 96-46. Bonn, 1996.

[3] V.B. Derjagin, A.N. Leznov, E. A. Yuzbashyan. Two-dimensional integrable mappings and explicit form of equations of $(1+2)$ dimensional hierarchies of integrable systems. Preprint MPI 96-39. Bonn, 1996.

[4] В.Е. Захаров, С.В. Манаков, С.П. Новиков, Л.П. Питаевский. Теория солитонов: Метод обратной задачи рассеяния. М.: Наука, 1980; Л. А. Тахтаджсян, Л. Д. Фаддеев. Гамильтонов подход в теории солитонов. М.: Наука, 1986; P. G. Olver. Application of Lie groups to differential equations. Berlin: Springer, 1986.

[5] А.Н. Лезнов. ТМФ. 1980. Т. 42. С. 343-349.

[6] A. N. Leznov. In: Proc. of II International Workshop "Nonlinear and turbulent process". Kiev, 1983. Ed. R. Sagdeev. New York: Gordon and Breach, 1984. P. 1437-1453.

[7] A. N. Leznov, V. G. Smirnov. Lett. Math. Phys. 1981. V. 5. P. 31-36.

[8] A. N. Leznov. In: Proc. of International Seminar "Group Methods in Physics", Zwenigorod, 24-26 November 1982. Ed. M. A. Markov. New York: Gordon and Breach, 1983. P. 443-457.

[9] A. N. Leznov, M. V. Saveliev. Progr. in Phys. 1992. V. 15. P. 290.

[10] A. Davey, K. Stewartson. Proc. Roy. Soc. 1974. V. A338. P. 101-110.

[11] A. N. Leznov, E. A. Yuzbashjan. Multi-soliton solution of two-dimensional matrix Davey-Stewartson equation. Preprint MPI-96-37. Bonn, 1996; Lett. Math. Phys. 1995. V. 35. P. $345-349$.

[12] А.Н. Лезнов, И. А. Федосеев. ТМФ. 1982. Т. 53. № 3. С. 358-373; I. A. Fedoseev, A. N. Leznov. Phys. Lett. В. 1984. V. 141. № 1-2. P. 100-103; А. Н. Лезнов, М. А. Мухтаров. ТMథ. 1987. T. 71. № 1. C. 46-53. 